\title{
THE BEGINNINGS OF THE SIX MILLION DOLLAR MAN (ORTHOPAEDIC IMPLANTS AND WHAT THEY ARE MADE OF)
}

\author{
A Feldman, Consultant Orthopaedic Surgeon \\ Royal Lancaster Infirmary
}

Orthopaedic surgeons started to use artificial materials to reconstruct bones and joints in the 1880s. Earlier attempts had been relatively unsuccessful, limited by the anaesthetic techniques then available and most importantly by a lack of knowledge regarding the need for antisepsis and cleanliness to avoid infection.

All surgery carries risk of infection and this risk is at its greatest when foreign or artificial material is implanted into the body. It was not until the institution of Joseph Lister's antiseptic techniques in 1870 s that implant surgery stood any chance of success.

The first and most obvious use for artificial implants was to hold together broken bones. Metal carpentry screws and engineering wires were first used, and then in 1890 William Arbuthnot Lane introduced dedicated metal plates (not dissimilar to Meccano plates) for the repair of longbone fractures. All this was massively aided by the discovery of Xrays in 1895 by Wilhelm Konrad von Roentgen. Now the bony fractures could be visualised and better repairs carried out using an ever-increasing number of metal implants.

The early orthopaedic implants were made of iron and various mild steels, and though they held the broken bone fragments for a while, they inevitably corroded and broke in the very hostile chemical environment of the body leading to increased problems rather than benefit.

The development of stainless steels, better plate designs and improvement to screw thread contours eventually led to implants that corroded much more slowly and were much less likely to break or work loose until the bone had a chance to heal.

As materials improved orthopaedic surgeons started to experiment with all sorts of potentially useful implants, and materials to realign broken bones, including the long rod-like intramedullary nails and various bone pins to be linked onto external fixation frames. Other implants were for the correction of developmental deformities in the skeletons of children and adults, such as spinal curvatures (scolioses) or to realign dislocated or deteriorating hip joints by osteotomy (cutting and realigning of bone).

Then in the late 1920 s it was suggested that it might be possible completely to replace arthritic joints that had been destroyed by trauma, inflammatory arthritis or, most commonly, degenerative osteoarthrosis. Such diseases most frequently affect the hip and knee joints, leading to exquisite pain, loss of mobility and loss of independence.

The first experiments involved the hip joint. In the mid 1920s Ernest William Hey Groves implanted several human hip replacements fashioned from elephant ivory (chosen for its similarity to bone), apparently with a modicum of success.
Obviously this was not practical on a large scale: currently over one million hip replacements are carried out worldwide every year, over one hundred thousand in the UK alone!

During the next thirty years British, American and French orthopaedic surgeons experimented intensely trying to produce a practical, reliable, reproducible and longlasting artificial total hip replacement. The best of these were made of stainless steel, cobalt-chrome alloy or acrylic plastic.

The real breakthroughs came in the 1960s when surgical teams in Wigan, Norwich, London and Exeter produced successful designs. Equally importantly, they developed relatively safe, repeatable surgical techniques to implant them. The surgeon acclaimed throughout the world as the true father and inventor of successful total hip replacement surgery was John Charnley at Wrightington Hospital, near Wigan. His design was made of stainless steel, polyethylene plastic and acrylic plastic cement.

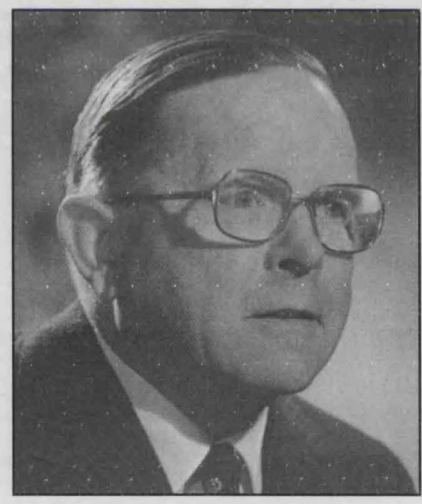

Sir John Charnley

Image courtesy of the John Charnley Trust

It is now some forty years since John Charnley implanted his first hip replacements. Surgeons from around the world flocked to Lancashire to learn the technique, and nowadays over 90 percent of hip replacements are successful, lasting 10 years or more, making this one of the most beneficial surgical operations yet to be invented. Currently there are in excess of fifty hip replacement designs used throughout the UK as surgeons try to improve the procedure even further.

Success with the hip joint naturally encouraged the development of other artificial joints. The past 20 years have seen equally successful knee replacement surgery, indeed many studies are suggesting that the knee replacement may be even more reliable than the hip replacement. In the UK we are now performing approximately equal numbers of knee and hip replacements each year.

Artificial joint replacements for the shoulder, elbow, wrist, ankle and some of the small joints in the hands and feet have now been developed. These are not as widely used as 
replacement hips and knees, as other kinds of medical and surgical treatment are often similarly successful.

In the UK today at least one in every twenty people has an artificial orthopaedic implant somewhere in their skeleton!

Modern surgical implants are made out of very sophisticated materials. The material chosen for each component of an implant must be:

\section{- mechanically suitable for its intended purpose}

- durable - implants are often required to last very many years (ideally they should outlive the person into whom they are implanted) without deterioration. This is extremely demanding as, for example, a hip or knee joint will undergo approximately one million cycles of movement and loading per week in an averagely active person. The loads passed through the joints in gentle walking are around four times body weight and considerably more during heavier activities

- biocompatible - the material must not be poisonous to, or adversely affect the health of, the person into whom it is implanted. The material must not be rejected by the body's immune system.

Three main groups of materials are used to manufacture orthopaedic implants: metals, plastics and ceramics.

\section{METALS}

Stainless steel is used in approximately $50 \%$ of the metal orthopaedic implants in current use across the world. There are a number of different stainless steel alloys employed, but a typical chemical composition for a stainless steel fracture fixation plate is shown in Figure 1.

\begin{tabular}{|l|l|}
\hline carbon $0.03 \%$ & nickel $14 \%$ \\
\hline chromium $18 \%$ & nitrogen $0.1 \%$ \\
\hline copper $0.25 \%$ & phosphorus $0.025 \%$ \\
\hline iron $62 \%$ & sulphur $0.01 \%$ \\
\hline manganese $1.5 \%$ & silicon $1 \%$ \\
\hline molybdenum $3 \%$ & \\
\hline
\end{tabular}

Figure 1

Cobalt chrome alloys are used in approximately $25 \%$ of metal orthopaedic implants and make exceptionally good mechanical joint bearings. The usual composition is around $65 \%$ cobalt, $30 \%$ chromium and $5 \%$ molybdenum. This is also known by the trade name of vitallium.

Titanium and titanium alloys are softer than the other metals and can be very useful for a number of orthopaedic situations. They are used in approximately $25 \%$ of metal orthopaedic implants. Titanium may be used in its pure form or alloyed with $6 \%$ aluminium and $4 \%$ vanadium to make it more durable.

Tantalum is used as a coating on the surface of a few very special orthopaedic implants as it is highly biocompatible with bone.

\section{PLASTICS}

High-density polyethylene (HDPE) is currently used to manufacture one side of the bearing surface in the majority of joint replacements. It has a very low coefficient of friction yet is extremely tough and long lasting. Originally John Charnley had experimented with another plastic, poly-tetra-fluoroethylene (PTFE or Teflon), which has a lower coefficient of friction but was completely unsuitable as it rapidly disintegrated under load.

Polyethylene was adopted for use in joint replacement bearings following its astonishing success in the manufacture of gear wheels used in the Lancashire cotton spinning and weaving industry in the1950s.

Poly-methyl-methacrylate or acrylic plastic (also known by the trade name 'perspex') is used as the cement in all cemented joint replacements. It is mixed to form a liquid during the surgical operation and takes between ten and fifteen minutes to polymerise and set into a solid. It does not glue the artificial joint to the bone but rather acts as a grout, filling the space between the bone and prosthesis. The first such orthopaedic bone cement was manufactured in Blackpool.

Some of the latest fracture fixation implants are being made from the absorbable plastics polylactic acid and polyglycolic acid. The body's phagocytic cells are able gradually to degrade and digest these plastics so that once the broken bone has healed these pins and screws completely vanish!

Silicone rubber (or poly-siloxane) is a very durable, biocompatible synthetic rubber. It is used to manufacture some of the tiny artificial joints used to rebuild hand and foot joints.

\section{CERAMICS}

Two ceramic materials, alumina (aluminium oxide) and zirconia (zirconium oxide) are used to manufacture very hard and very smooth joint bearings for hip joints. Theoretically they should have a longer life expectancy than the more commonly used metal bearings but they are brittle and delicate with a tendency to crack or shatter if abnormally loaded.

Hydroxyapatite ceramic is a very exciting substance. It is basically made out of the same material as the mineral component of bones, calcium-hydroxy phosphate. The body can be tricked into thinking that implants made of this material, or more often coated with it, are in fact its own bones. Consequently, new bone is built solidly onto the artificial prosthesis as the body heals.

Carbon fibre is another new material being used in orthopaedic surgery. It can be used alone as a woven mesh to support healing cartilage or as a fibre reinforcement within a polymer plastic from which extremely tough yet slightly flexible fracture fixation plates can be made.

Britain has led the world in the development of orthopaedic implants and continues very much at the forefront of this technology. Forty years ago relatively little could be done to alleviate the disability and pain of major skeletal injury, disease and arthritis. Today, over two hundred thousand patients in Britain each year receive a new joint or undergo the rebuilding of their skeletons by orthopaedic surgery employing artificial implants. 